\title{
Positive Predictive Value of ICD-I0 Diagnosis Codes for COVID-19
}

\author{
Jacob Bodilsen (1D' \\ Steffen Leth $\mathbb{D}^{2,3}$ \\ Stig Lønberg Nielsen (iD) 4,5 \\ Jon Gitz Holler 6 \\ Thomas Benfield (iD ${ }^{7}$ \\ Lars Haukali Omland (iD ${ }^{8}$ \\ 'Department of Infectious Diseases, \\ Aalborg University Hospital, Aalborg, \\ Denmark; ${ }^{2}$ Department of Infectious \\ Diseases, Aarhus University Hospital \\ Skejby, Aarhus, Denmark; ${ }^{3}$ Department \\ of Medicine, Regional Hospital Unit West \\ Jutland, Herning, Denmark; ${ }^{4}$ Research \\ Unit for Infectious Diseases, Odense \\ University Hospital, Odense, Denmark; \\ ${ }^{5}$ University of Southern Denmark, \\ Odense, Denmark; ${ }^{6}$ Department of \\ Pulmonary and Infectious Diseases, \\ Hillerød Hospital, Hillerød, Denmark; \\ ${ }^{7}$ Department of Infectious Diseases, \\ Copenhagen University Hospital - \\ Amager and Hvidovre, Hvidovre, \\ Denmark; ${ }^{8}$ Department of Infectious \\ Diseases, Copenhagen University \\ Hospital, Rigshospitalet, Copenhagen, \\ Denmark
}

Purpose: To examine the positive predictive value (PPV) of International Classification version 10 (ICD-10) diagnosis codes for Coronavirus disease 2019 (COVID-19).

Patients and Methods: Medical record review of all patients assigned a diagnosis code of COVID-19 (DB342A or DB972A) at six Danish departments of infectious diseases from February 27 through May 4, 2020. Confirmed COVID-19 diagnosis was defined as either: 1) definite, a positive polymerase chain reaction (PCR) for severe acute respiratory syndrome Coronavirus 2 (SARS-CoV-2) on a respiratory sample combined with symptoms suggestive of COVID-19: 2) probable, clinical presentation of COVID-19 without detection of SARSCoV-2 and no alternative diagnoses considered more likely; or 3) possible, clinical presentation of COVID-19 without detection of SARS-CoV-2, and the patient was discharged or deceased before further investigations were carried out. We computed the PPV with 95\% confidence intervals $(\mathrm{CI})$ as the number of patients with confirmed (i.e., definite, probable, and possible) COVID-19 divided by the number of patients assigned a diagnosis code for COVID-19.

Results: The study included 710 patients with a median age of 61 years (interquartile range [IQR] 47-74) and 285/710 (40\%) were female. COVID-19 was confirmed in 706/710 (99\%) with 705/710 (99\%) categorized as definite, $1 / 710(0.1 \%)$ as probable, and 0 patients as possible COVID-19. The diagnosis was disproven in $4 / 710(0.6 \%)$ patients who were hospitalized due to bacterial pneumonia $(\mathrm{n}=2)$, influenza $(\mathrm{n}=1)$, and urinary tract infection $(\mathrm{n}=1)$. The overall PPV for COVID-19 was 99\% (95\% CI 99-100) and remained consistently high among all subgroups including sex, age groups, calendar period, and stratified by diagnosis code and department of infectious diseases (range 97-100\%).

Conclusion: The overall PPV of diagnosis codes for COVID-19 in Denmark was high and may be suitable for future registry-based prognosis studies of COVID-19.

Keywords: Coronavirus disease 2019; COVID-19, SARS-CoV-2, diagnosis codes, ICD-10, positive predictive value; PPV, validation, epidemiology

\section{Plain-Language Summary}

We reviewed the medical records of 710 patients admitted at six departments of infectious diseases in Danish hospitals from February 27 through May 4, 2020 with an ICD-10 diagnosis code of Coronavirus disease (COVID-19) and found an overall positive predictive value (PPV) of 99\%. The results were consistent across different diagnosis codes for COVID-19, age groups, sex, calendar period, and departments of infectious diseases. These results are important for ensuring the validity of studies using ICD-10 diagnosis codes to identify COVID-19 patients.
Correspondence: Jacob Bodilsen Department of Infectious Diseases, Aalborg University Hospital, Mølleparkvej 4, Aalborg, 9000, Denmark

Email Jacob.bodilsen@rn.dk 


\section{Introduction}

COVID-19 is a potentially life-threatening infection for aging and other vulnerable populations. ${ }^{1-4}$ Health care databases allow researchers to effectively explore both common and rare associations in large populations, ${ }^{5-10}$ and may be valuable for long-term monitoring of efficiency and safety of treatments and vaccines for COVID-19 outside the settings of randomized controlled trials. In addition, the epidemiological characteristics of COVID-19 are dynamic and rapidly change within and between countries as the pandemic evolves, and large health care registries may assist in quickly testing scientific hypothesis in different settings. ${ }^{11-15}$ However, registrybased analyses using diagnosis codes require that the codes are of high quality and, thus far, the validity of ICD-10 codes for COVID-19 remains unclear. This study aimed to examine the PPV of the ICD-10 diagnosis codes for COVID-19 at departments of infectious diseases in Denmark.

\section{Methods}

\section{Setting}

In Denmark, medical care is tax-supported and free of charge at the point of delivery for all residents. A unique civil registration number assigned at birth or immigration allows for the unique identification of all Danish residents and unambiguous linkage between registries. ${ }^{7}$

\section{Study Design and Study Population}

This cross-sectional validation study was conducted by reviewing the medical records of all patients admitted with a first-time ICD-10 diagnosis code for COVID-19 (DB342A and DB972A) at departments of infectious diseases (and affiliated "pandemic departments") at hospitals in Aalborg, Aarhus, Odense, Hillerød, and Copenhagen University Hospitals at Amager/Hvidovre and Rigshospitalet in Denmark from February 27 through May 4, 2020 (Figure 1). Patients were identified by searches at each department and the diagnosis codes correspond to those that are reported to the Danish National Patient Registry. ${ }^{9}$

\section{Record Review and Definition of COVID- 19}

During medical record review, the admission date of firsttime hospitalization for COVID-19 was used as index date. If patients were tested positive for Severe acute respiratory syndrome Coronavirus 2 (SARS-CoV-2) while hospitalized for other reasons, the date of test for SARS-CoV-2 was considered the index date. Next, a local investigator reviewed the medical records of all hospitalized patients assigned a COVID-19 diagnosis code at each center including doctor's notes, laboratory results, microbiological analyses, imaging results as described by the

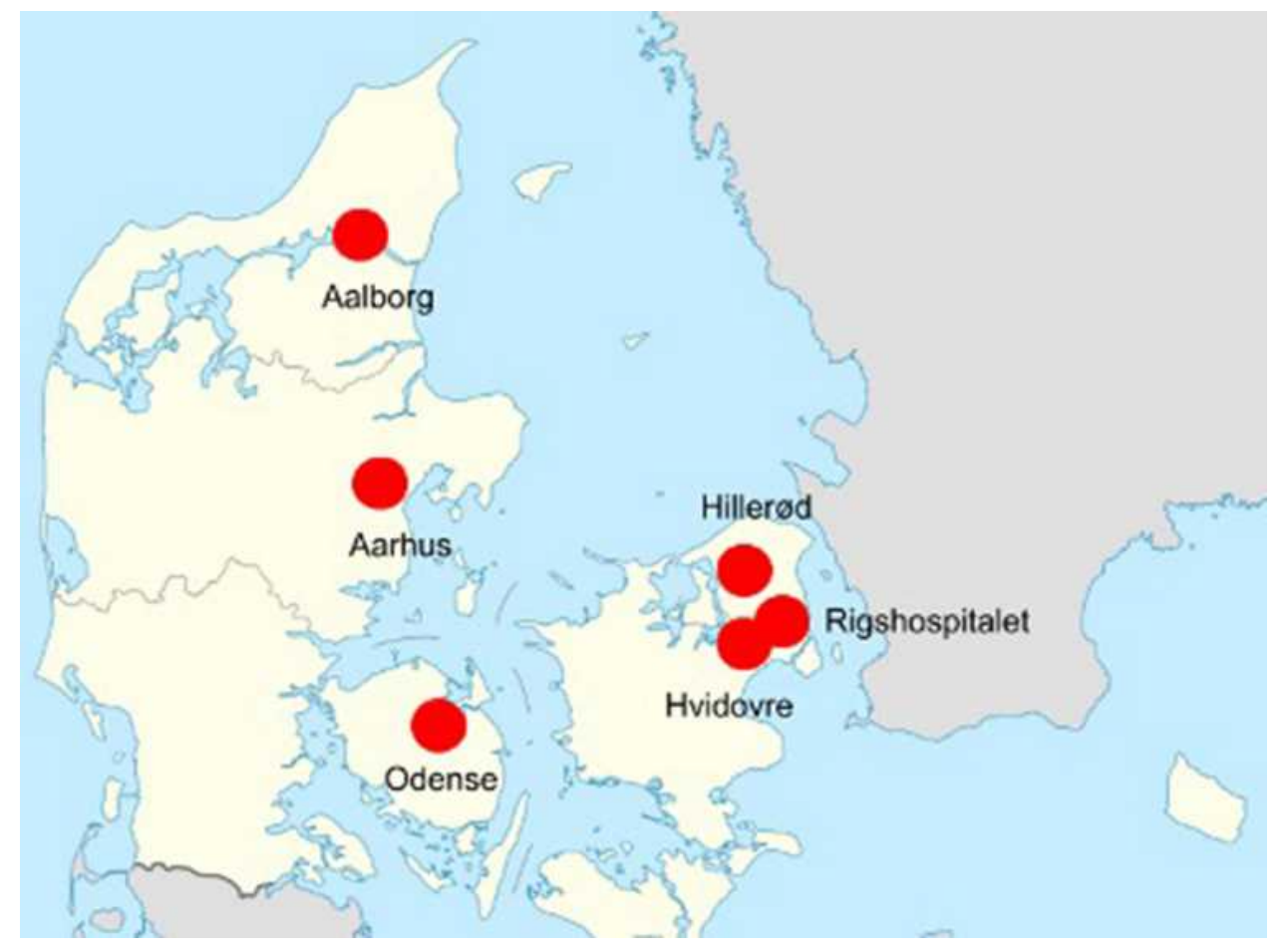

Figure I Geographical distribution of the involved departments of infectious diseases in Denmark. 
hospital radiologist, and outcomes of the patients. In cases of doubt, categorizations of patients were resolved by discussion (JB and LO). The following definitions for COVID-19 were used:

Definite COVID-19:

1. A positive polymerase chain reaction (PCR) for SARS-CoV-2 on a respiratory sample AND

2. A clinical presentation consistent with COVID-19, e. g., fever, sore throat, headache, nasal congestion, dyspnea, cough, nausea/vomiting, diarrhea, or myalgia.

Probable COVID-19:

1. A clinical presentation consistent with COVID-19, i. e., any combination of fever, sore throat, headache, nasal congestion, dyspnea, cough, nausea/vomiting, diarrhea, and myalgia without detection of SARS-CoV-2 on a respiratory sample AND

2. No other pathogen detected, and no other medical condition was considered more likely.

Possible COVID-19:

1. A clinical presentation consistent with COVID-19, $\mathrm{i}$. e., any combination of fever, sore throat, headache, nasal congestion, dyspnea, cough, nausea/vomiting, diarrhea, and myalgia without detection of SARS-CoV-2 on a respiratory sample AND

2. The patient passed away or was discharged before further examinations had been performed including tests for SARS-CoV-2 and no other medical condition was considered more likely.

A diagnosis of COVID-19 was considered disproven if the patient did not fulfill any of the criteria listed above or had a confirmed alternative diagnosis.

\section{Statistical Analyses}

The PPV was calculated as the number of confirmed (i.e., definite, probable, and possible) COVID-19 cases divided by the number of patients assigned a diagnosis code of COVID-19 and using medical record review combined with results of PCR test for SARS-CoV-2 as reference. Using the exact binomial method, the PPV with 95\% confidence intervals (CIs) was computed for patients with confirmed COVID-19 and by each category of definite, probable, and possible COVID-19. Additional analyses of the PPV for definite COVID-19 were conducted stratified by diagnosis code (DB342A or DB972A), age groups (0-40, 41-60, 61-80, 81+ years), sex, and calendar period (February 27 through March 31, and April 1 through May 4). A post hoc analyses considering only definite diagnosis of COVID-19 for assessment of the PPV was also carried out. Categorical variables are presented as $\mathrm{n} / \mathrm{N}(\%)$ and continuous variables as medians with interquartile ranges (IQR).

Study data were collected and managed using REDCap electronic data capture tools hosted at North Denmark Region. ${ }^{16}$ Stata/MP ${ }^{\circledR}$ version 16 (StataCorp LLC, Texas) was used for all statistical analyses.

\section{Ethical Considerations}

The study was approved by the legal department at the North Denmark Region (record number 2020-045) and the Danish Board of Health (record number 31-1522-84). Patient consent or permission from an ethical committee is not required for this type of study in Denmark. Handling of data complied with relevant data protection and privacy regulations and was conducted in accordance with the Helsinki declaration.

\section{Results}

During the study period, a total of 710 patients were assigned a diagnosis code of COVID-19 (Table 1). The median age of patients was 61 years (IQR 47-74) and 285/710 (40\%) were females. Consistent with the overall development of the first wave of the pandemic in Denmark, $542 / 710(76 \%)$ patients were admitted early during the study period from February 27 through March 31, 2020.

A definite diagnosis of COVID-19 was observed in 705/ $710(99 \%)$ of patients, whereas the diagnosis was probable in $1 / 710(0.1 \%)$ and possible in 0 patients (Table 2). COVID-19 was disproven in $4 / 710(0.6 \%)$ patients, of which 2 were diagnosed with bacterial pneumonia (both had unknown pathogens and a clinical response to antibiotic therapy), 1 with influenza, and 1 with a urinary tract infection.

Using both definite and probable diagnoses of COVID19 as reference, the overall PPV for COVID-19 was 99\% (95\% CI 99-100) compared with 99\% (95\% CI 98-100) when using definite cases only (Table 3). The PPV was consistently high and ranged between $97 \%$ and $100 \%$ among all subgroups including sex, age groups, calendar period, and stratified by diagnosis code (DB342A and DB972A) and department of infectious diseases.

\section{Discussion}

This study observed a very high PPV of COVID-19 diagnosis codes for patients hospitalized in Denmark during 
Table I Baseline Characteristics of 710 Patients Assigned a FirstTime ICD-10 Diagnosis Code for COVID-19 at Departments of Infectious Diseases in Denmark from February 27 Through May 4, 2020

\begin{tabular}{|l|c|}
\hline Characteristic & n (\%) or Median (IQR) \\
\hline Diagnosis codes & $566(80)$ \\
DB342A & $144(20)$ \\
DB972A & \\
\hline Sex & $285(40)$ \\
Females & $425(60)$ \\
Males & $61(47-74)$ \\
\hline Age group, years & $116(16)$ \\
0-40 & $234(33)$ \\
4 I-60 & $263(37)$ \\
6I-80 & $97(14)$ \\
8 I- & \\
\hline Calendar period & $542(76)$ \\
February 27 through March 3I, 2020 & $168(24)$ \\
April I through May 4, 2020 & \\
\hline Department of infectious diseases & $78(11)$ \\
Aalborg & $67(9)$ \\
Aarhus & $75(11)$ \\
Hillerød & $83(12)$ \\
Odense & $314(44)$ \\
Hvidovre/Amager & $93(13)$ \\
Rigshospitalet & \\
\hline
\end{tabular}

Abbreviation: IQR, interquartile ranges.

Table 2 Distribution of Confirmed COVID-19 Among Patients Assigned ICD-I0 Diagnosis Codes for COVID-19 (DB342A and DB972A) at Departments of Infectious Diseases in Denmark

\begin{tabular}{|c|c|c|}
\hline COVID-19 & Number of Observations (n/N) & $\%$ \\
\hline Confirmed & $706 / 710$ & 99 \\
Definite & $705 / 710$ & 99 \\
Probable & $1 / 710$ & 0.1 \\
Possible & 0 & 0 \\
\hline Disproved & $4 / 710$ & 0.6 \\
\hline
\end{tabular}

the first wave of the pandemic. The PPV ranged 97-100\% in all examined subgroups including sex, age groups, and when stratified by diagnosis code.

The ongoing COVID-19 pandemic has led to an impressive and unprecedented concerted effort of the entire global scientific community to rapidly explore the characteristics and treatment of this infection resulting in more than 100,000 scientific publications on the topic in year $2020 .{ }^{17}$ Databases using ICD-10 diagnosis codes may be a valuable tool in expanding existing knowledge on COVID-19 by analyzing associations in large-scale populations. ${ }^{7-9,18}$ However, it remains pivotal that the accuracy of the used diagnosis codes is high. Using SARS-CoV-2 test positivity as reference, a large registry-based study from a US administrative all-payer repository observed a PPV of 92\% (95\% CI 91-92) for ICD-10 diagnosis codes for COVID-19. ${ }^{19}$ In general, the validity of diagnosis codes in Danish health care databases is high, ${ }^{9}$ and the results of the current study provide assurance of the usefulness of ICD-10 codes for COVID-19 in Denmark.

Previous registry-based studies have often utilised large microbiological databases of SARS-CoV-2 test-positive and test-negative persons to explore scientific hypothesis related to COVID-19. Strengths of this approach include capture of the majority of individuals with proven infection and identification of a potential control population (i.e., SARS-CoV-2 test negative persons). However, a large proportion of SARSCoV-2 positive individuals may have asymptomatic infection $^{20-24}$ and the current study may thus allow researchers to combine clinical disease characteristics of COVID-19 with documented SARS-CoV-2 infection.

This study has limitations. Selection bias may have been introduced by changes in testing strategies and management of COVID-19 patients. However, all patients admitted to hospital with symptoms of COVID-19 were tested for SARS-CoV-2 throughout the study period in Denmark. In addition, the study included all patients assigned a diagnosis code of COVID-19 at the participating centers representing different geographic regions of Denmark. Ascertainment bias, i.e., researchers examining the medical records were aware of the diagnosis code beforehand and may have favored confirmation of COVID-19 in cases of doubt, was mitigated by accessing all available information in the medical records and adhering to a strict a priori definition of confirmed COVID-19. Moreover, $99 \%$ of patients had relevant symptoms and tested positive for SARS-CoV-2 by PCR of a respiratory sample. The study was not able to test the sensitivity, specificity, or completeness of the ICD-10 diagnosis codes for COVID-19, because only patients assigned such diagnosis codes were examined.

The generalizability of the results of this study is improved by the large sample size including all patients with a COVID-19 diagnosis code at participating centers representative of a country with free health care for all residents. Still, coding practices may differ from noninfectious diseases departments and other health care settings, or during later stages of the pandemic. 
Table 3 Positive Predictive Value (PPV) with 95\% Confidence Intervals (CI) of ICD-I0 Diagnosis Codes for COVID-I9 (DB342A and DB972A) at Departments of Infectious Diseases in Denmark

\begin{tabular}{|c|c|c|}
\hline COVID-19 & Number of Observations $(n / N)$ & PPV \% $(95 \% \mathrm{Cl})$ \\
\hline Confirmed overall & $706 / 710$ & $99(99-100)$ \\
\hline Definite cases only & $705 / 710$ & $99(98-100)$ \\
\hline \multicolumn{3}{|l|}{ Diagnosis codes } \\
\hline DB342A & $564 / 566$ & $100(99-100)$ \\
\hline DB972A & $142 / 144$ & $99(95-100)$ \\
\hline \multicolumn{3}{|l|}{ Sex } \\
\hline Females & $283 / 285$ & $99(97-100)$ \\
\hline Males & $423 / 425$ & $100(98-100)$ \\
\hline \multicolumn{3}{|l|}{ Age group } \\
\hline $0-40$ & $116 / 116$ & $100(97-100)$ \\
\hline $4 I-60$ & $233 / 234$ & $100(98-100)$ \\
\hline $61-80$ & $261 / 263$ & $99(97-100)$ \\
\hline $81-$ & $96 / 97$ & $99(94-100)$ \\
\hline \multicolumn{3}{|l|}{ Calendar period } \\
\hline February 27 through March 3I, 2020 & $540 / 542$ & $100(99-100)$ \\
\hline April I through May 4, 2020 & $166 / 168$ & $99(96-100)$ \\
\hline \multicolumn{3}{|l|}{ Department of infectious diseases } \\
\hline Aalborg & $76 / 78$ & $97(9 \mid-100)$ \\
\hline Aarhus & $67 / 67$ & $100(95-100)$ \\
\hline Hillerød & $75 / 75$ & $100(95-100)$ \\
\hline Odense & $83 / 83$ & $100(96-100)$ \\
\hline Hvidovre/Amager & $3|3 / 3| 4$ & $100(98-100)$ \\
\hline Rigshospitalet & $92 / 93$ & $99(94-100)$ \\
\hline
\end{tabular}

In conclusion, the overall PPV of ICD-10 diagnosis codes for COVID-19 was very high and may be suitable for future registry-based prognosis studies of COVID-19.

\section{Abbreviations}

CI, Confidence interval; COVID-19, Corona virus disease 2019; ICD-10, International Classification of Diseases versions 10; PPV, Positive predictive value; SARS-CoV-2, Severe acute respiratory syndrome coronavirus 2 .

\section{Disclosure}

The authors report no conflicts of interest in this work.

\section{References}

1. Reilev M, Kristensen KB, Pottegård A, et al. Characteristics and predictors of hospitalization and death in the first 11122 cases with a positive RT-PCR test for SARS-CoV-2 in Denmark: a nationwide cohort. Int J Epidemiol. 2020;49(5):1-14. doi:10.1093/ije/dyaa140

2. Zhou F, Yu T, Du R, et al. Clinical course and risk factors for mortality of adult inpatients with COVID-19 in Wuhan, China: a retrospective cohort study. Lancet. 2020;395(10229):1054-1062. doi:10.1016/ S0140-6736(20)30566-3
3. Goyal P, Choi JJ, Pinheiro LC, et al. Clinical characteristics of Covid-19 in New York City. New Engl J Med. 2020;382 (24):2372-2374. doi:10.1056/NEJMc2010419

4. Woolf SH, Chapman DA, Lee JH. COVID-19 as the leading cause of death in the United States. JAMA. 2021;325(2):123-124. doi:10.1001/jama.2020.24865

5. Sørensen HT, Pedersen L, Jorgensen J, Ehrenstein V. Danish clinical quality databases - an important and untapped resource for clinical research. Clin Epidemiol. 2016;8:425-427. doi:10.2147/CLEP. S113265

6. Johannesdottir SA, Horváth-Puhó E, Ehrenstein V, Schmidt M, Pedersen L, Sørensen HT. Existing data sources for clinical epidemiology: the Danish National Database of Reimbursed Prescriptions. Clin Epidemiol. 2012;4(1):303-313. doi:10.2147/ CLEP.S37587

7. Schmidt M, Pedersen L, Sørensen HT. The Danish Civil Registration System as a tool in epidemiology. Eur J Epidemiol. 2014;29 (8):541-549. doi:10.1007/s10654-014-9930-3

8. Schmidt M, Schmidt SAJ, Adelborg K, et al. The Danish health care system and epidemiological research: from health care contacts to database records. Clin Epidemiol. 2019;11:563-591. doi:10.2147/ CLEP.S179083

9. Schmidt M, Schmidt SAJ, Sandegaard JL, Ehrenstein V, Pedersen L, Sørensen HT. The Danish National Patient Registry: a review of content, data quality, and research potential. Clin Epidemiol. 2015;7:449-490. doi:10.2147/CLEP.S91125

10. Frank L. Epidemiology: when an entire country is a cohort. Science. 2000;287(5462):2398-2399. doi:10.1126/science.287.5462.2398 
11. Pottegård A, Kristensen KB, Reilev M, et al. Existing data sources in clinical epidemiology: the Danish COVID-19 Cohort. Clin Epidemiol. 2020;12:875-881. doi:10.2147/CLEP.S257519

12. Lund LC, Kristensen KB, Reilev M, et al. Adverse outcomes and mortality in users of non-steroidal anti-inflammatory drugs who tested positive for SARS-CoV-2: a Danish nationwide cohort study. Plos Med. 2020;17(9):e1003308. doi:10.1371/journal.pmed.1003308

13. Pottegård $\mathrm{A}$, Kurz $\mathrm{X}$, Moore $\mathrm{N}$, Christiansen $\mathrm{CF}$, Klungel $\mathrm{O}$. Considerations for pharmacoepidemiological analyses in the SARS-CoV 2 pandemic. Pharmacoepidemiol Drug Saf. 2020;29 (8):825-831. doi:10.1002/pds.5029

14. Christiansen CF, Pottegård A, Heide-Jørgensen U, et al. SARS-CoV -2 infection and adverse outcomes in users of ACE inhibitors and angiotensin-receptor blockers: a nationwide case-control and cohort analysis. Thorax. 2020. doi:10.1136/thoraxjnl-2020-215768

15. Dalager-Pedersen M, Lund LC, Mariager T, et al. Venous thromboembolism and major bleeding in patients with COVID-19: a nationwide population-based cohort study. Clin Infect Dis. 2021; ciab003.

16. Harris PA, Taylor R, Thielke R, Payne J, Gonzalez N, Conde JG. Research electronic data capture (REDCap) - A metadata-driven methodology and workflow process for providing translational research informatics support. $J$ Biomed Inform. 2009;42 (2):377-381. doi:10.1016/j.jbi.2008.08.010
17. Else H. How a torrent of COVID science changed research publishing — in seven charts. Nature. 2020;588(7839):553. doi:10.1038/ d41586-020-03564-y

18. Schneeweiss S, Avorn J. A review of uses of health care utilization databases for epidemiologic research on therapeutics. $J$ Clin Epidemiol. 2005;58(4):323-337. doi:10.1016/j.jclinepi.2004.10.012

19. Kadri SS, Gundrum J, Warner S, et al. Uptake and accuracy of the diagnosis code for COVID-19 among US hospitalizations. JAMA. 2020;324(24):2553-2554. doi:10.1001/jama.2020.20323

20. Sakurai A, Sasaki T, Kato S, et al. Natural history of asymptomatic SARS-CoV-2 infection. New Engl J Med. 2020;383(9):885-886. doi:10.1056/NEJMc2013020

21. Wiersinga WJ, Rhodes A, Cheng AC, Peacock SJ, Prescott HC. Pathophysiology, transmission, diagnosis, and treatment of Coronavirus disease 2019 (COVID-19). JAMA. 2020;324 (8):782-793. doi:10.1001/jama.2020.12839

22. Gandhi M, Yokoe DS, Havlir DV. Asymptomatic transmission, the Achilles' Heel of current strategies to control Covid-19. New Engl $J$ Med. 2020;382(22):2158-2160. doi:10.1056/NEJMe2009758

23. Rothe C, Schunk M, Sothmann P, et al. Transmission of 2019-nCoV infection from an asymptomatic contact in Germany. New Engl $J$ Med. 2020;382(10):970-971. doi:10.1056/NEJMc2001468

24. Oran DP, Topol EJ. Prevalence of asymptomatic SARS-CoV-2 infection: a narrative review. Ann Intern Med. 2020;173(5):362-367. doi:10.7326/M20-3012
Clinical Epidemiology

\section{Publish your work in this journal}

Clinical Epidemiology is an international, peer-reviewed, open access, online journal focusing on disease and drug epidemiology, identification of risk factors and screening procedures to develop optimal preventative initiatives and programs. Specific topics include: diagnosis, prognosis, treatment, screening, prevention, risk factor modification, systematic reviews, risk \& safety of medical interventions, epidemiology \& biostatistical methods, and evaluation of guidelines, translational medicine, health policies \& economic evaluations. The manuscript management system is completely online and includes a very quick and fair peer-review system, which is all easy to use.

\section{Dovepress}

